\section{Chikungunya Disease Outbreak, Reunion Island}

To the Editor: A serious outbreak of chikungunya disease recently occurred on Reunion Island (population $\approx 770,000$ ) (1). Between March 1 , 2005, and April 30, 2006, $\approx 255,000$ cases were reported in this French territory in the Indian Ocean. Most cases occurred after mid-December 2005, with a maximum of 45,000 cases during the week of January 29 to February 4, 2006 (2). Surveillance figures were confirmed by a serosurvey that found a prevalence of $18 \%$ of recent infection markers in pregnant women in March 2006.

Chikungunya is a self-limiting febrile viral disease characterized by arthralgia or arthritis. Symptoms may last for several months, but recovery was, until now, considered universal (3). However, in January 2006, the health authorities on this island started receiving death certificates mentioning chikungunya as a cause of death, either direct or indirect. By the end of April, 213 death certificates with this finding had been received. To assess the affect of chikungunya disease, we compared the crude death rate (CDR) observed during the outbreak period with an expected death rate computed from the 2002-2004 historical data.

The study included the period January 1, 2005, through April 30, 2006. The expected number of deaths (all causes) for 2005 and 2006 was the number of deaths by sex and age observed during 2002-2004 modified by an estimation of the population size for 2005-2006. The details of this method, which was used during the heat wave in France in 2003, have been reported (4). The number of deaths in Reunion was obtained daily from 13 of 24 computerized registry offices throughout the island and represented $87 \%$ of the deaths on the island.
During 2005, the monthly CDR remained within expected range of statistical variation. From January through April 2006, respectively, monthly CDRs were $7.1 \%, 34.4 \%$, $25.2 \%$, and $8.3 \%$ higher than expected rates $(p<0.01$ for February and March). This corresponded to 226 excess deaths reported by the 13 offices participating in the study and 260 excess deaths when data were extrapolated to the entire population of the island (an increase of 18.4\%) (Figure). The 260 excess deaths is a crude figure that includes potentially all causes of death. This figure leads to a rough estimate of the case-fatality rate for chikungunya disease of $\approx 1 / 1,000$ cases. Excess deaths were observed mainly in persons $\geq 75$ years of age.

CDRs began to exceed the expected range during the last week of January 2006 and remained elevated until the end of the study period. This situation closely matched the kinetics of the epidemic curve of chikungunya disease. CDR is a stable variable in time for a defined population. Only a massive phenomenon can have an effect on it, and no other abnormal health event affected the island at this time. Thus, the outbreak of chikungunya disease was likely responsible for most of the excess deaths observed in Reunion during the first 4 months of 2006.

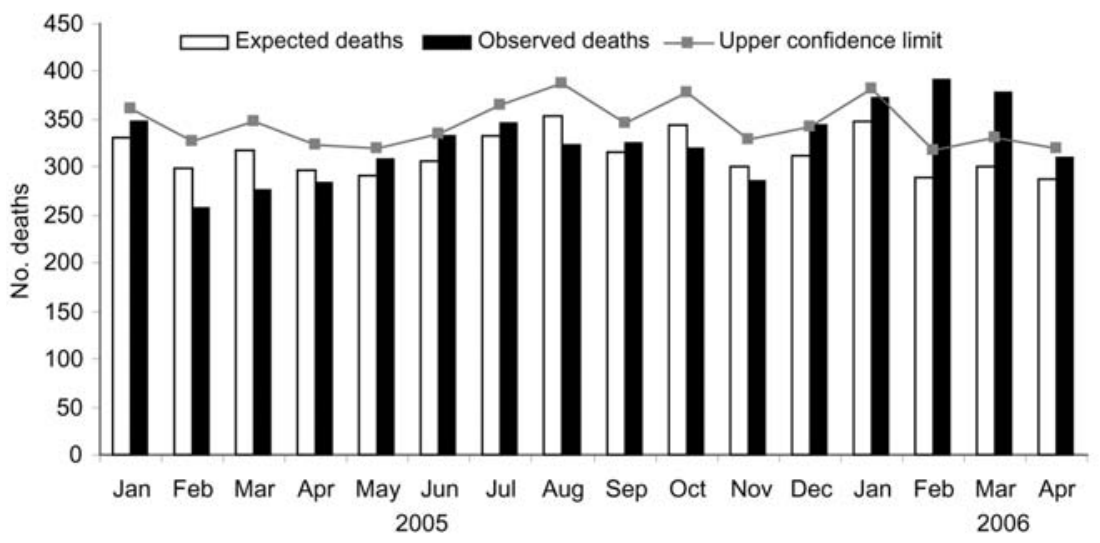

Figure. Expected and observed number of deaths reported by 13 computerized registry offices in Reunion Island, France, January 2005-April 2006. 
4. Pirard P, Vandentorren S, Pascal M, Laaidi K, Le Tertre A, Cassadou S, et al. Summary of the mortality impact assessment of the 2003 heat wave in France. Euro Surveill. 2005;10:153-6.

Address for correspondence: Loïc Josseran, French National Institute for Public Health Surveillance, Institut de Veille Sanitaire, 12, Rue du Val d'Osne, 94415 Saint Maurice CEDEX, France; email: 1.josseran@invs. sante.fr

\section{Assay to Detect H5N 1 Oseltamivir Resistance}

To the Editor: Oseltamivir is a neuraminidase inhibitor approved for treatment and prevention of influenza virus infection. Oseltamivir resistance caused by a single amino acid substitution from histidine $(\mathrm{H})$ to tyrosine (Y) at position 274 of the neuraminidase active site has been reported in persons infected both experimentally and naturally with influenza A virus subtype H5N1 $(1,2)$. Evidence suggests that using lower doses of oseltamivir or shorter-than-recommended treatment periods can contribute to emergence of viral resistance $(1,3)$ Currently, oseltamivir is being used in several countries that may be affected by epidemics of H5N1. Therefore, monitoring for oseltamivir-resistant strains of H5N1 during oseltamivir administration is essential for outbreak management and prevention.

Although real-time PCR or pyrosequencing is more rapid than high-throughput assays for mutation detection $(4,5)$, the conventional PCR technique can be applied to detect drug-resistant mutation (6) in areas lacking real-time PCR or pyrosequencing capabilities. Therefore, to discriminate between oseltamivirsensitive and oseltamivir-resistant strains, we developed a simple method, based on PCR, which takes advantage of the H274Y substitution. The forward primer was designed from the conserved region common to both wild-type and mutant strains; the reverse primers were designed specifically for wild-type and mutant strains, respectively, derived from the 3 ' terminal base of each primer. The primers consisted of a forward primer N1f (nt 517-534: 5'-GGGGCTGTGGCTGTATTG-3') and reverse primer H274r (nt 759-784: 5'-GGATAACAGGAGCAYTCCTCATAGTG -3') for wild-type strain detection or Y274r (nt 759-784: 5'-GGATAACAGGAGCAYTCCTCATAGTA-3') for mutant strain detection. (Note: Underlined letters represent differences in nucleotides between plus vs. minus primers.) Both strains yielded products of $\approx 267 \mathrm{bp}$; hence, the assay consisted of 2 separate reactions for detecting wild-type and mutant strains, respectively.

For each reaction, $1.0 \mu \mathrm{L}$ cDNA was combined with a reaction mixture that contained $10 \mu \mathrm{L} 2.5 \times$ MasterMix (Eppendorf, Hamburg, Germany), forward and reverse primers at a final concentration of $0.15 \mu \mathrm{M}$, and nuclease-free water to a final volume of 20 $\mu \mathrm{L}$. Thermocycling conditions comprised initial denaturation at $94^{\circ} \mathrm{C}$ for 3 min and 35 cycles of amplification including denaturation $\left(94^{\circ} \mathrm{C}, 30 \mathrm{~s}\right)$, annealing $\left(65^{\circ} \mathrm{C}, 50 \mathrm{~s}\right)$, extension $\left(72^{\circ} \mathrm{C}, 45 \mathrm{~s}\right)$, and final extension $\left(72^{\circ} \mathrm{C}, 7 \mathrm{~min}\right)$. Subsequently, $10 \mu \mathrm{L}$ of the amplified products was analyzed by using $2 \%$ agarose gel electrophoresis.

To optimize the assay, we performed PCR-based H274Y mutagenesis of the N1 fragment of the H5N1 virus (primers on request). The resulting mutagenic and wild-type products were cloned into the pGEM-T Easy Vectors (Promega, Madison, WI, USA), confirmed by direct sequenc- ing, and then used as positive controls. Preliminary results showed that the wild-type primer was specific for the oseltamivir-sensitive strain, whereas the mutant primer can be used to detect the oseltamivir-resistant strain exclusively because no significant cross-amplification had been observed.

To establish sensitivity, serial 10fold dilutions of the standard N1 plasmids (wild-type and mutant) ranging from $10^{9}$ to $10^{1}$ copies/ $\mu \mathrm{L}$ were used as a template. The threshold concentrations for detection of wild-types and mutants were $10^{3}$ copies $/ \mu \mathrm{L}$. To provide semiquantitative data to detect subpopulations of the resistant variants, the 2 control plasmids were mixed at wild-type:variant ratios of $10^{8}: 10^{2}, 10^{7}: 10^{3}, 10^{6}: 10^{4}, 10^{5}: 10^{5}, 10^{4}$ : $10^{6}, 10^{3}: 10^{7}$, and $10^{2}: 10^{8}$. The result showed that the density of the expected bands depended on the amount of DNA templates (Figure B). However, the mixing experiments indicated that the predominant mixtures of wildtype:resistant variant were 80:20, which is the lowest ratio of resistant variants that the assay can reliably detect (data not shown). To assess specificity, human DNA and viral cDNA extracted from other subtypes of influenza A virus (N2-N9) were subjected to this assay. No cross-reaction occurred with human DNA or other subtypes of influenza A virus.

We further validated the assay by testing 3 specimens from hosts treated with oseltamivir and 17 specimens from untreated hosts; infection with H5N1 was detected by using multiplex real-time PCR (7). The specimens from oseltamivir recipients were isolated from a Vietnamese patient on the third day after he received a prophylactic dose (1) and from 2 tigers (CU-T7 and KU-11) (8). The specimens from untreated hosts were isolated from the plasma of an H5N1-infected human (9) and from virus-containing allantoic fluid isolated from infected chickens, ducks, 